\title{
Endoscopes for observation of plasma-wall interactions in the divertor of Wendelstein 7-X
}

\author{
Olaf Neubauer ${ }^{\mathrm{a}}$, Anton Charl ${ }^{\mathrm{a}}$, Guntram Czymek ${ }^{\mathrm{a}}$, Yu Gao ${ }^{\mathrm{a}}$, Martin Knaup ${ }^{\mathrm{a}}$, Ralf König ${ }^{\mathrm{b}}$, Maciej \\ Krychowiak $^{\mathrm{b}}$, Horst-Toni Lambertz ${ }^{\mathrm{a}}$, M. Lennartz ${ }^{\mathrm{c}}$, Christian Linsmeier ${ }^{\mathrm{a}}$, G. Satheeswaran ${ }^{\mathrm{a}}$, Bernd \\ Schweer ${ }^{\mathrm{d}}$, Mathias Schülke ${ }^{\mathrm{b}}$, Stepan Sereda ${ }^{\mathrm{a}}$, and the W7-X Team \\ ${ }^{a}$ Forschungszentrum Jülich GmbH, Institut für Energie- und Klimaforschung - Plasmaphysik, Partner of the Trilateral \\ Euregio Cluster (TEC), 52425 Jülich, Germany \\ ${ }^{b}$ Max Planck Institute for Plasma Physics, 17491 Greifswald, Germany \\ ${ }^{c}$ Forschungszentrum Jülich GmbH, Central Institute for Engineering, Electronics and Analytics - Plasmaphysik, 52425 \\ Jülich, Germany \\ ${ }^{d}$ Laboratoire de Physique des Plasmas - Laboratorium voor Plasmafysica, ERM/KMS, Partner of the Trilateral \\ Euregio Cluster (TEC), 1000 Brussels, Belgium
}

\begin{abstract}
The stellarator Wendelstein 7-X is being prepared for long pulse operation. This includes diagnostics for investigation of plasma wall interaction processes. A versatile optical observation system has been developed for local characterization of the divertor plasma and the divertor target surface. The optical systems consist of two endoscopes each with perpendicular fields of view and the opportunity of tomographic reconstruction. Mirror based optics has been chosen in order to assure good optical properties independent of the wavelength. A narrow field of view allows for high spatial resolution while rotation of the first mirror covers the full poloidal divertor sections. An integrated shutter mechanism and a vacuum window far back minimize coating of optical components. For assessment of change of light transmission, a relative calibration function is implemented. The output light is split into wavelength ranges. Both, cameras equipped with narrow band filters as well as spectrometers are connected. The first endoscope was mounted at W7-X after successfully passing mechanical, optical and functional tests.
\end{abstract}

Keywords: stellarator, Wendelstein 7-X, plasma wall interactions, diagnostic, endoscope, piezo drive

\section{Introduction}

The superconducting stellarator Wendelstein $7-\mathrm{X}$ is being prepared for long pulse operation with reactor relevant plasmas at steady state conditions [1-5] aiming to proof the optimized stellarator as serious candidate for a fusion reactor. Plasma Wall Interactions (PWI) play a crucial role in this respect and thus form an important field of research at Wendelstein 7-X. Key issues of PWI such as lifetime of plasma-facing components (PFC's), and tritium retention have to be fully understood in a fusion power plant.

For the most relevant PWI zone, Wendelstein 7-X is to test the concept of an island divertor. In particular, divertor plasma properties have to be studied in detail. The 3D topology of edge plasma together with steady state capability of Wendelstein $7-X$ provide the opportunity to investigate specific aspects of PWI which could contribute to answer the question of reactor relevance of a quasi-isodynamic stellarator configuration together with island divertor. However, this particular configuration provides a challenge for experimental investigation and modelling compared to more or less axisymmetric configurations.

With a strong focus and background on PWI research at several experiments, together with its preparation by diagnostic development, an experimental program on 'PWI in 3D Plasma Boundary at W7-X' is prepared by developing a dedicated set of diagnostic instruments and modelling activities [6,7]. Newly developed versatile endoscopes [8] for divertor observation being a major tool within this program are described in this paper.

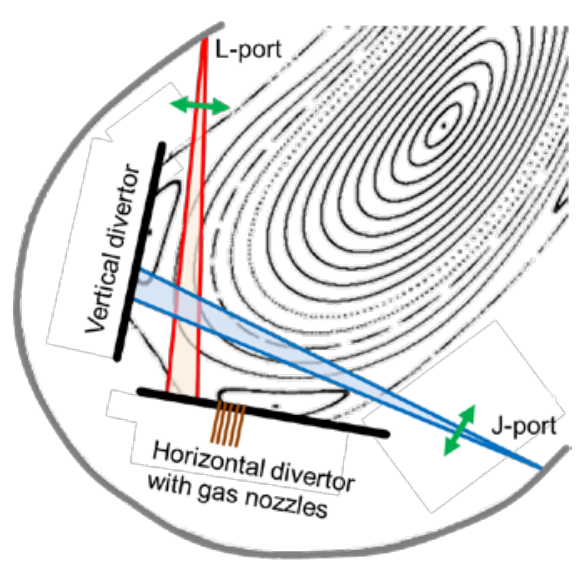

Fig. 1. Horizontal (J-port) and vertical (L-port) fields of view of first mirrors at the vacuum-vessel wall with respect to the divertor. A Poincare plot in standard configuration represents the plasma.

\section{Objectives}

The aim of the PWI diagnostic set is to characterize upstream and downstream parameters of the edge plasma and processes at the divertor surface. For this reason, a fast probe manipulator [9] and a poloidal correlation reflectometer [10] have already been developed and installed. A versatile optical observation system [8] in two machine modules located along the same magnetic 
structure has now been added. Together with two gas inlets installed in the region observed, temperature and density profiles, impurity transport and recycling, hydrogen recycling, and transient heat load deposition can be analysed.

\section{Concept}

The observation system consists of two endoscopes each with perpendicular fields of view (Fig. 1) with the opportunity of tomographic reconstruction. Mirror based optics with a vacuum window far back and cameras outside have been chosen for independence from wavelength, and in order to minimize coating of optical elements and neutron damage to windows and detectors, and finally to maximize flexibility.

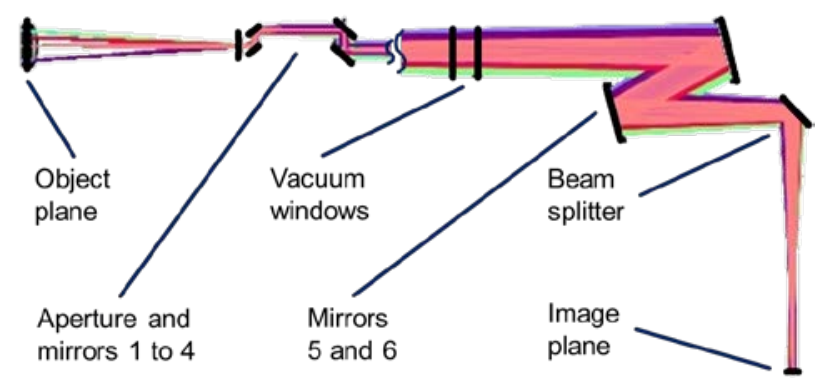

Fig. 2. Optical concept of the endoscopes (first of several beam splitters shown only).

The layout (Fig. 2) has been derived from endoscopes for JET [11] developed in the past with major improvements. A narrow field of view allows for high spatial resolution while rotation of the first mirror covers the full poloidal divertor sections. An aperture improves image quality and together with an integrated shutter mechanism in front of the first mirror reduces coating of optical components. For assessment of change of transmission, a relative calibration function is implemented. In a detector box a roof shaped mirror, dichroic beam splitters, and intensity splitters provide fractions of light to individual detectors. Six cameras partly equipped with narrow band filters and image intensifiers (in UV range only), and four fibre couplers for spectrometers are available simultaneously at each endoscope.
The main optical parameters to be achieved by the concept are listed in Table 1.

Table 1 Main parameters of the endoscopes.

\begin{tabular}{lll}
\hline & Unit & Value \\
\hline $\begin{array}{l}\text { Spectral range } \\
\text { ultraviolet } \\
\text { visible + infrared }\end{array}$ & $\mathrm{nm}$ & $350-550$ \\
infrared & $\mathrm{nm}$ & $550-900$ \\
$\begin{array}{c}\text { Transmittance } \\
\text { w/o splitters }\end{array}$ & $\%$ & $>300-7000$ \\
$\begin{array}{l}\text { Spatial resolution } \\
\text { Object distance }\end{array}$ & $\mathrm{mm}$ & $<2$ \\
$\begin{array}{l}\text { Depth of field } \\
\text { Horizontal/vertical } \\
\text { fields of view }\end{array}$ & $\mathrm{m}$ & $0.8-0.7$ \\
$\begin{array}{c}\text { Poloidal rotation } \\
\text { of first mirror }\end{array}$ & $\mathrm{m}$ & 0.6 \\
Aperture size & degrees & 9 \\
Object size & $\mathrm{mm} * \mathrm{~mm}$ & $4 * 4$ \\
\hline
\end{tabular}

The light collected by the first mirror (M1) in the vicinity of the vacuum-vessel wall has to be reflected via another mirror through a vacuum window outside the cryostat vessel to the rear optics. Rotation of M1 leads to image distortion which is almost perfectly compensated by a properly shaped second pair of mirrors in the endoscope front end. Two mirrors outside the cryostat provide the required focal length (Fig. 2).

\section{Design}

Apart from functional requirements, the design (Fig. 3) of the endoscopes as of any other diagnostic has to take into account restrictions given by the environment of Wendelstein 7-X such as material selection, microwave radiation, heat load, magnetic properties, compatibility with tight space, assembly, conditioning and service procedures [12].

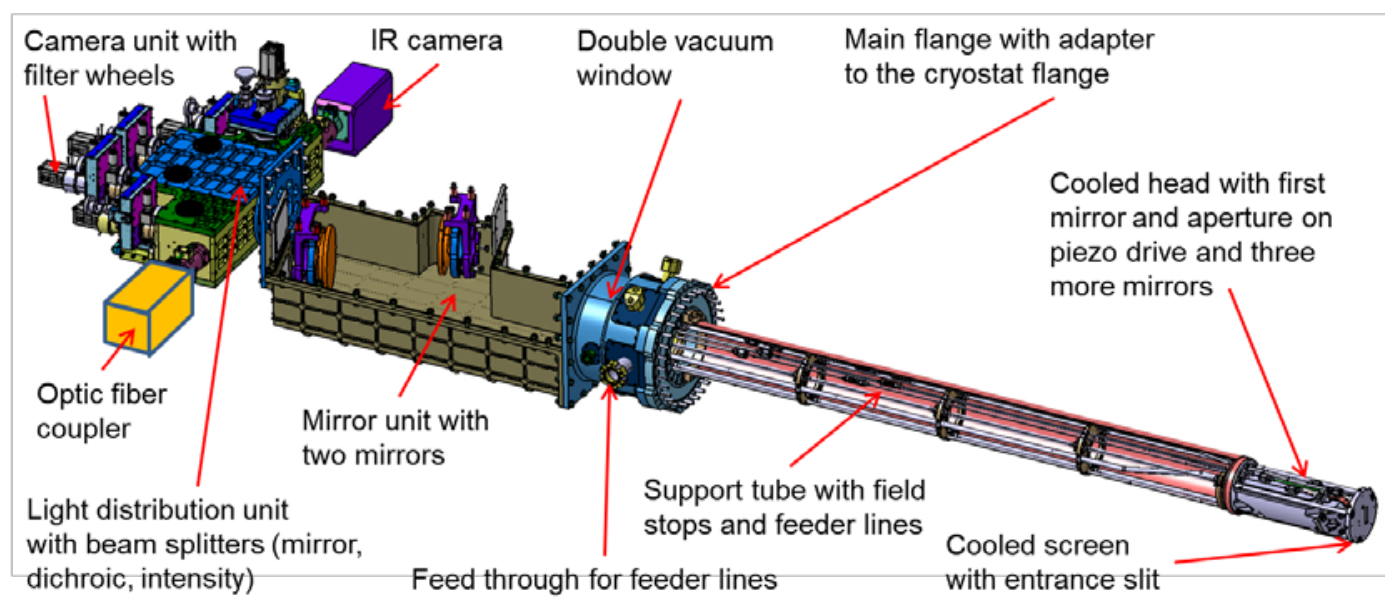

Fig. 3. Schematic view of the endoscope design. 
In particular, properly designed water-cooling of the head and front screen guarantee compatibility with full power steady state plasmas (radiation up to $100 \mathrm{~kW} / \mathrm{m}^{2}$ ). A narrow entrance slit in the screen together with the aperture in the head minimize penetration of microwave stray radiation into the endoscope.

The endoscope head (Fig. 4) contains four mirrors, the first one mounted in a casing with aperture and reflective coating on a piezoelectric drive. Rotation inside an outer casing combines functionalities of choosing field of view, shutter, and back reflection of calibration light. All mirrors have three-point supports for adjustment.

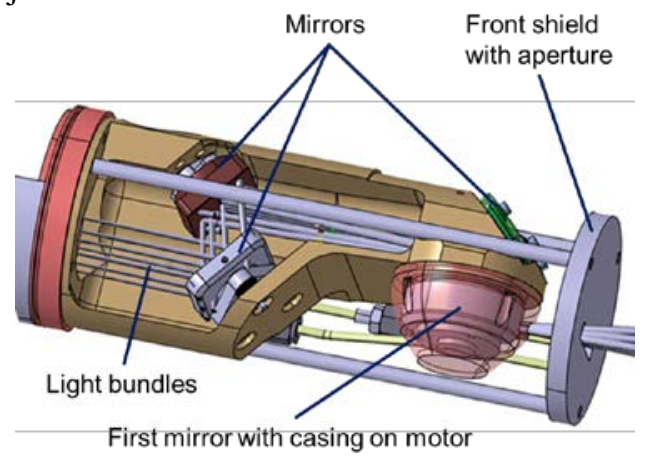

Fig. 4. Endoscope head with screen removed.

Several baffles in the support tube reduce stray light propagation. The tube flange transfers full weight and momentum of the endoscope to the cryostat flange. Wedge-shaped rings provide an angular adjustment compensating tolerances in the cryostat geometry.

A double vacuum window with interspace monitoring provides a safe vacuum barrier. $\mathrm{CaF}_{2}$ is sufficiently transparent over the required wavelength range. Wedge-like shaping of the windows deflects ghost images out of the field of the subsequent mirror, however requires careful mutual adjustment.

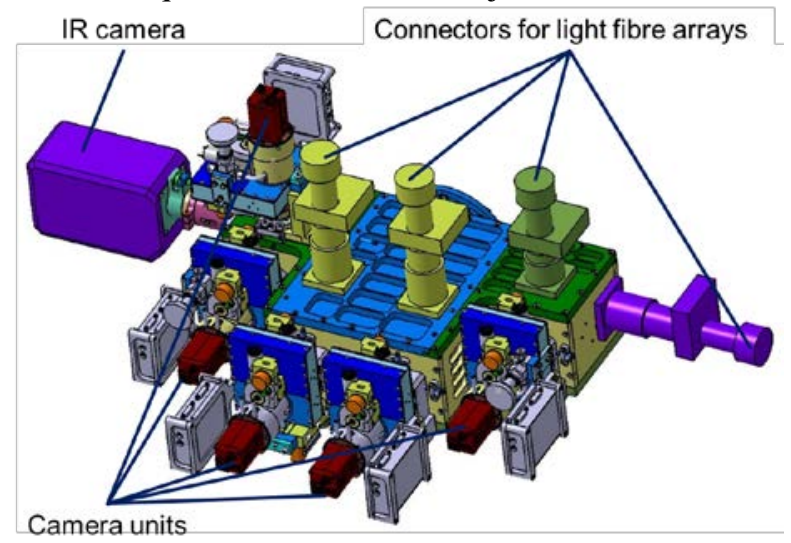

Fig. 5. Light distribution unit.

The mirror unit at atmosphere contains two mirrors on angularly adjustable mounts and is followed by the light distribution unit (Fig. 5) with several beam splitters (roof shaped mirror, dichroic beam splitter, intensity splitter) in order to supply individual detectors.

Camera units (Fig. 6) with adjustable mounts consist of a CCD sensor mounted on a Peltier cooler, a taper for adaption of image size, an image intensifier (for UV range only), two filter wheels with 6 narrow band interference filters or 6 attenuation filters, respectively. Control of filter selection and high voltage for image intensifier is integrated into the camera control unit. Five units in total allow for simultaneous observation of two lines in the UV range and another three in the visible.

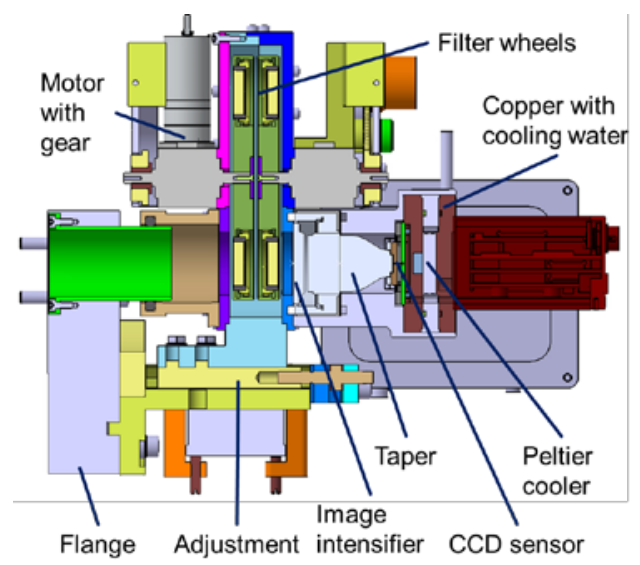

Fig. 6. Camera unit.

A commercial camera with integrated filters sits on one of the IR outputs. Optical fibres route several IR, UV and visible outputs to spectrometers. Piezo motors rotate the couplers (Fig. 7) synchronously to the first mirror in order to maintain image orientation.

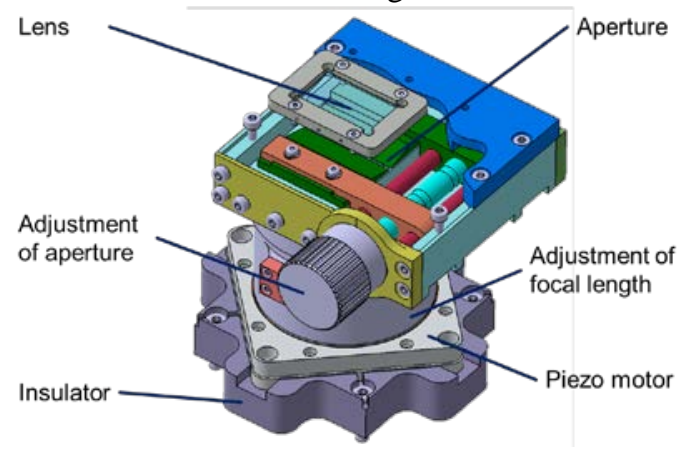

Fig. 7. Connector for light fibre array.

In contrast to the outputs of the light distribution unit, optical fibres integrated into the edge of the roof shaped mirror at the pupil of the light path deliver light integrated over the field of view to an overview spectrometer. At the same location, another fibre provides back illumination of the mirrors towards the first one for relative intensity calibration.

The overall optical and mechanical design enables to rotate components along the light path without image distortion. Thus in particular the distribution unit can be rotated to fit into the tight space available at W7-X.

\section{Manufacturing, assembly, and testing}

The first endoscope for J-port in sector 51 is complete. As expected for an optical system, manufacturing tolerances of mirrors and supports, and structural interfaces of all components required particular attention. 
A first check of the optical path after assembly delivered a focussed image (Fig. 8) proving successful chain of data from optical via mechanical design, manufacturing and assembly with sufficient precision. Moreover, disassembly of the endoscope into three units (support tube with flange and window, mirror unit, and distribution unit) as required for mounting at an inner port of Wendelstein 7-X with limited access and reassembly without loss of adjustment was proven.

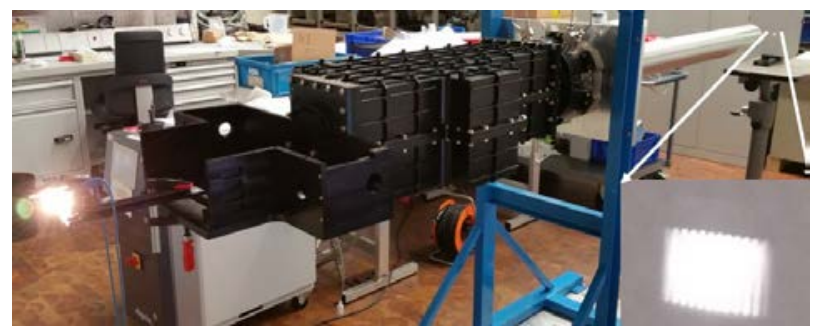

Fig. 8. First optical test with light source (left) produced a focussed image on a screen (right).

Quality assurance put a dedicated focus on welding seams at vacuum and cooling water boundaries. Corresponding welding procedures were qualified in advance.

The total weight well below $200 \mathrm{~kg}$ matching the load bearing capability of the cryostat flange and the centre of gravity close to the flange facilitating mounting of the endoscope at the torus and minimizing torque on the flange have been achieved.

A comprehensive test program has been performed before delivery including test of:

- Outgassing and leakage

- Material properties, welding seams

- Handling of the endoscope

- Disassembly into three parts and reassembly

- Function of mirror rotation, filter wheels, image intensifiers, cameras

- Optical transmission, optical resolution
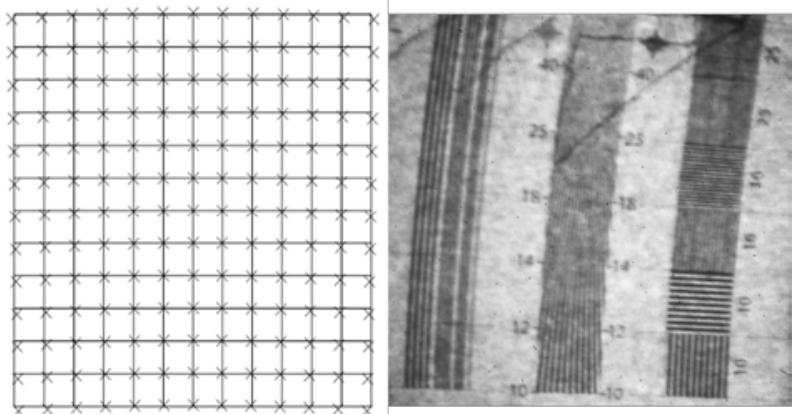

Fig. 9. Simulated image distortion (marks on regular grid, left) and real image of a square shaped figure with lines at varying distance seen by camera in visible range with excellent spatial resolution (numbers indicate line pairs per $\mathrm{cm}$ ) but considerable distortion.

Figures 9 and 10 represent results of optical tests. Both prove spatial resolution better than specified. However, a considerable image distortion was found which is attributed to insufficient mirror alignment. This will be improved by adjusting each mirror starting from the image plane to point at the optical center of the subsequent one.
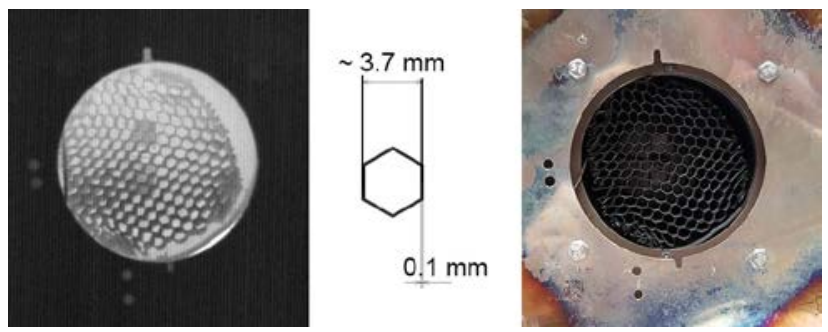

Fig. 10. Image seen by IR camera (left) and photograph (right) of cold honeycomb structure in front of a heated plate $\left(350^{\circ} \mathrm{C}\right)$.

\section{Conclusions and outlook}

The investigation of plasma wall interactions of Wendelstein 7-X requires local highly resolved observation of plasma and plasma facing surfaces in the divertor region. A new endoscope considerably improved with respect to the one formerly developed for JET has successfully been designed, manufactured and tested. New features are rotation of the first mirror including shutter and back reflection functions, image correction close to the first mirror, and long pulse compatibility.

Currently the L-port endoscope for sector 51 is under manufacturing. Geometric boundary conditions lead to a first mirror and therefore the endoscope head closer to the plasma. Design has been adapted with the results of dedicated heat load analysis. Together with another pair of endoscopes for sector 30 of Wendelstein 7-X, the diagnostic will be fully functional for the first long pulse campaign with water-cooled divertor.

\section{Acknowledgment}

The authors appreciate excellent assembly work by Thomas Krings and Karl von Bovert.

This work has been carried out within the framework of the EUROfusion Consortium and has received funding from the Euratom research and training programme 2014-2018 under grant agreement No 633053. The views and opinions expressed herein do not necessarily reflect those of the European Commission.

\section{References}

[1] Th. Klinger et al., IAEA 2018

[2] Th. Sunn Pedersen et al., IAEA 2018

[3] H.-S Bosch et al., Nucl. Fusion 57 (2017) 116015

[4] R. C. Wolf et al., Nucl. Fusion 57 (2017) 102020

[5] Th. Sunn Pedersen et al., Physics of Plasma 24 (2017) 055503

[6] Y. Liang et al., Nucl. Fusion 57 (2017) 066049

[7] O. Neubauer et al., Fus. Eng. Design 96-97 (2015) 891894

[8] O. Neubauer et al., PoS (ECPD2015) 113

[9] D. Nicolai et al., Fus. Eng. Design 123 (2017) 960-964

[10] A. Krämer-Flecken et al., Nucl. Fusion 57 (2017) 066023

[11] A. Huber, A., Rev. Sci. Instrum. 83, 10D511 (2012)

[12] D. Hathiramani, Fus. Eng. Design, in press 